COMECHINGONIA. REVISTA DE ARQUEOLOGÍA

Número 14, 2011, pp. 115-131, Córdoba

ISSN 0326-7911

\title{
CARACTERIZACIÓN GEOAMBIENTAL Y CULTURAL DEL PERÍODO FORMATIVO EN SELVAS OCCIDENTALES MERIDIONALES: SITIO “HORCO MOLLE” (DPTO. YERBA BUENA, TUCUMÁN)
}

\author{
Mario G. Maldonado(1-2), Liliana del V. Neder ${ }^{(2)}$, Jimena Roldán ${ }^{(1-2)}$ y \\ María M. Sampietro Vattuone $e^{(1-2)}$
}

(1)CONICET. (2)Laboratorio de Geoarqueología, Facultad de Ciencias Naturales e I.M.L, Universidad Nacional de Tucumán (UNT). Av Alem 114. San Miguel de Tucumán, CP 4000. gabrielmaldonado23@yahoo.com.ar

Presentado el: 19/10/2010 - Aceptado 20/03/2011

\section{Resumen}

El objetivo del presente trabajo es contribuir al conocimiento de las características geoambientales y culturales del piedemonte tucumano durante el Período Formativo y las formas en que los grupos humanos interactuaron con ellas. La metodología articuló fotointerpretación geomorfológica, excavaciones arqueológicas, descripción de perfiles de suelos y análisis tipológico del material cerámico y lítico. Concluimos que condiciones paleoclimáticas húmedas y suelos loéssicos favorecieron el asentamiento de grupos formativos con cerámica de estilo Candelaria sobre unidades geomorfológicas diferentes (abanico aluvial y glacis cubierto) durante las Fases Chuscha (200-400 DC) y Choromoro (400-700 DC), y posiblemente Molleyaco (400-700 DC) y Rupachico (700-1000 DC). La disponibilidad y adecuación de ciertas rocas y minerales para la producción de artefactos líticos y cerámicos influyeron en bajos costos de obtención, de manufactura, en los procesos de depositación, escaso reuso y en la ausencia de reclamación de artefactos. Los resultados son de interés para la arqueología de Selvas Occidentales meridionales.

Palabras claves: Asentamientos del Período Formativo, Sitios arqueológicos Candelaria, Selvas Occidentales Meridionales.

Abstract

The goal of this paper is to contribute to the knowledge of geoenvironmental and cultural characteristics of Tucumán's piedmont during Formative Period, together with the reconstruction of the ways in which human groups interacted with those characteristics. Geomorphological photointerpretation, archaeological digs, soil profile descriptions, and typological analysis of lithic and ceramic materials were made. We conclude that wet paleoclimatic conditions and loessic soils favored Formative settlements with Candelaria ceramic style over alluvial fans and covered glacis geomorphological units. Those settlements belonged to Chuscha (200-400 DC) and Choromoro (400700 DC) phases, and possible Molleyaco (400-700 DC) and Rupachico (700-1000 DC) phases. The availability and quality of some rocks and minerals for lithic and ceramic production influenced low 
obtaining costs, low manufacturing costs, fast depositional processes, scarce reuse, and low artifact claim. Our results are interesting for southern Selvas Occidentales archaeology.

Keywords: Formative Period Settlements, Candelaria Archaeological Sites, Southern Selvas Occidentales.

\section{Introducción}

En el NOA el sector de las Selvas Occidentales presenta escaso conocimiento arqueológico, lo que se acentúa en la región que va desde el dique El Cadillal hacia el sur siguiendo el piedemonte tucumano. En ese espacio se conoce escasamente cómo los sistemas socioculturales formativos interactuaron con el entorno geoambiental (Butzer 1989), a diferencia de los valles intermontanos adyacentes (Sampietro et al. 2003). El objetivo general de este trabajo es contribuir al conocimiento de dichos aspectos, mediante investigaciones en el sitio "Horco Molle" del piedemonte oriental de la Sa. de San Javier (Dpto. Yerba Buena, Tucumán), asignado a la "cultura Candelaria" (Heredia 1975 entre otros) (figura 1).

Presentamos el análisis y caracterización del contexto geomorfológico y pedológico, los contextos arqueológicos excavados y los artefactos líticos y cerámicos recuperados, analizando la influencia general de la disponibilidad y adecuación de las materias primas para su producción. Se aborda la cronología relativa usando la secuencia de Heredia (1975), la que aún se toma como referencia (Scattolin 2007), aunque con reservas.

\section{Antecedentes}

Para el Período Formativo de Selvas Occidentales meridionales se conoce lo que se ha denominado "cultura Candelaria" (Ryden 1936, Heredia 1975, entre otros), incluyendo al

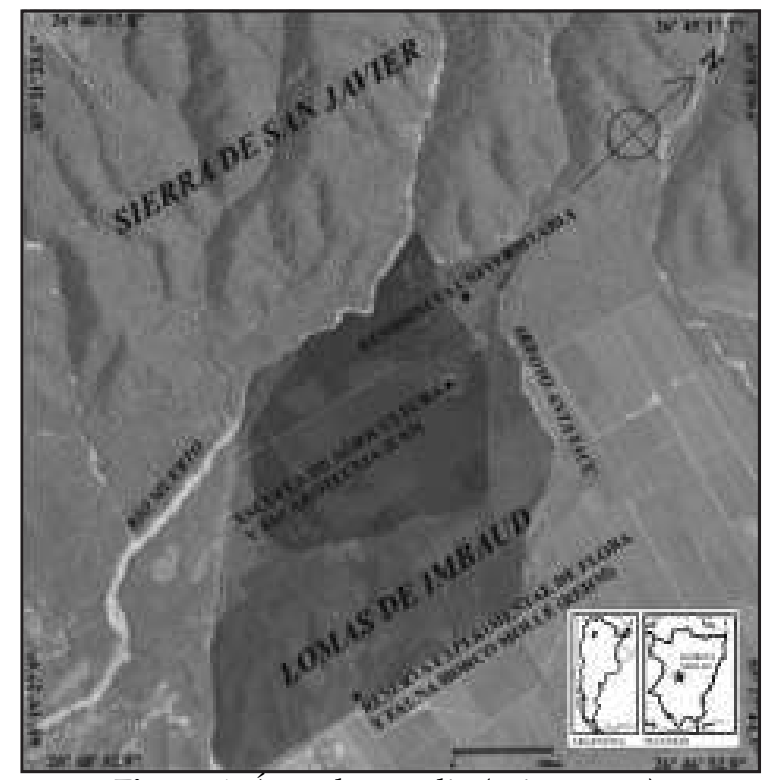

Figura 1. Área de estudio (gris oscuro). 
piedemonte de la Sa. de San Javier y a Horco Molle (Angiorama et al. 1992, Martínez et al. 1994, entre otros). Candelaria quedó definida como "cultura" principalmente en base a la dispersión de su cerámica, dada la carencia de datos de otros elementos del "contexto cultural". La idea de que a cada subárea o región del NOA correspondían determinadas culturas generó que Heredia considerara que Candelaria sólo pertenecía a Selvas Occidentales, idea que limita el entendimiento de la dinámica cultural regional (Scattolin et al. 2007). La secuencia cronológica de Heredia (1975) ha sido criticada para sus momentos I, IV y V (Núñez Regueiro y García Azcárate 1996, Tartusi y Núñez Regueiro 2000, Srur 2001) quedando a salvo de observaciones críticas los momentos II, III. Se conocen en total 19 fechados radiocarbónicos para contextos formativos de Selvas Occidentales meridionales (Ventura 2003). La relación de estos grupos humanos con el entorno ambiental se ha entendido de modo general y con referencia a la subárea de Selvas Occidentales como unidad espacial amplia, y es escaso y poco detallado su entendimiento considerando ambientes más específicos dentro del ámbito selvático, o considerando la "textura geoambiental".

Las investigaciones arqueológicas del Período Formativo para el piedemonte de la Sa. de San Javier y Horco Molle no escapan a las problemáticas mencionadas. Se ha sugerido una cronología relativa para el sitio (Martínez et al. 1994, Cano 2003) pero no se efectuaron estudios específicos sobre el tema. Casi no se han caracterizado rigurosamente los artefactos hallados ni se han estudiado contextos preservados. Se ha propuesto un uso del espacio geomorfológico basado en una percepción socialmente construida de espacios domésticos y sagrados (Míguez 2005), aunque con menor peso en factores geoambientales.

\section{Metodología}

Para caracterizar morfogenéticamente a Horco Molle se realizó fotointerpretación (escala 1:20.000) siguiendo los criterios de Van Zuidam y Van Zuidam (1985). Se tomó como unidad espacial de estudio un abanico aluvial enmarcado por el río Muerto y el arroyo Anta Yacu, y se excavó en 7 sectores, describiendo a campo los rasgos macromorfológicos de perfiles pedológicos (horizontes, estructura, textura, limites, etc.) (Etcheverre 1976).

Los fragmentos cerámicos se clasificaron en grupos macroscópicos (Primera Convención de Antropología 1966) distinguiendo entre decorados (técnicas decorativas) y no decorados (grupos de pastas, acabado de superficie), estableciendo finalmente los tipos. Se obtuvieron y compararon frecuencias porcentuales para los atributos y tipos cerámicos distinguidos y se estableció la presencia/ ausencia de los tipos decorados, reconocidos en nuestro conjunto dentro de las fases de la seriación de Heredia (1975). Se analizó la distribución estratigráfica de frecuencias de acabados de superficies y decoración en relación a la alteración de los fragmentos cerámicos por procesos de arrastre hídrico, considerando el tamaño de los fragmentos (< a $1 \mathrm{~cm} ; 1,1$ a $3 \mathrm{~cm} ; 3,1-6 \mathrm{~cm} ; 6,1-9 \mathrm{~cm}$; > a $9 \mathrm{~cm}$ ), forma (angulosos, subredondeados/subangulosos, redondeados) y redondeo de bordes (alto, bajo, o no erodados) (Maldonado et al. 2009).

El material lítico fue clasificado de acuerdo con la propuesta de Aschero $(1975,1983)$, distinguiendo en el conjunto variedades de materias primas, separando entre: desechos de talla (Estado e Índice de Fragmentación, Número Mínimo de Desechos, dimensiones, origen de las extracciones, ancho de talones y tipos de talones); núcleos; artefactos formatizados y con rastros complementarios (enteros vs. fracturados, tipo de forma base, dimensiones, serie 
técnica y grupo tipológico). La tipología cerámica y lítica permitió vislumbrar la influencia general de la disponibilidad de ciertas materias primas para su producción.

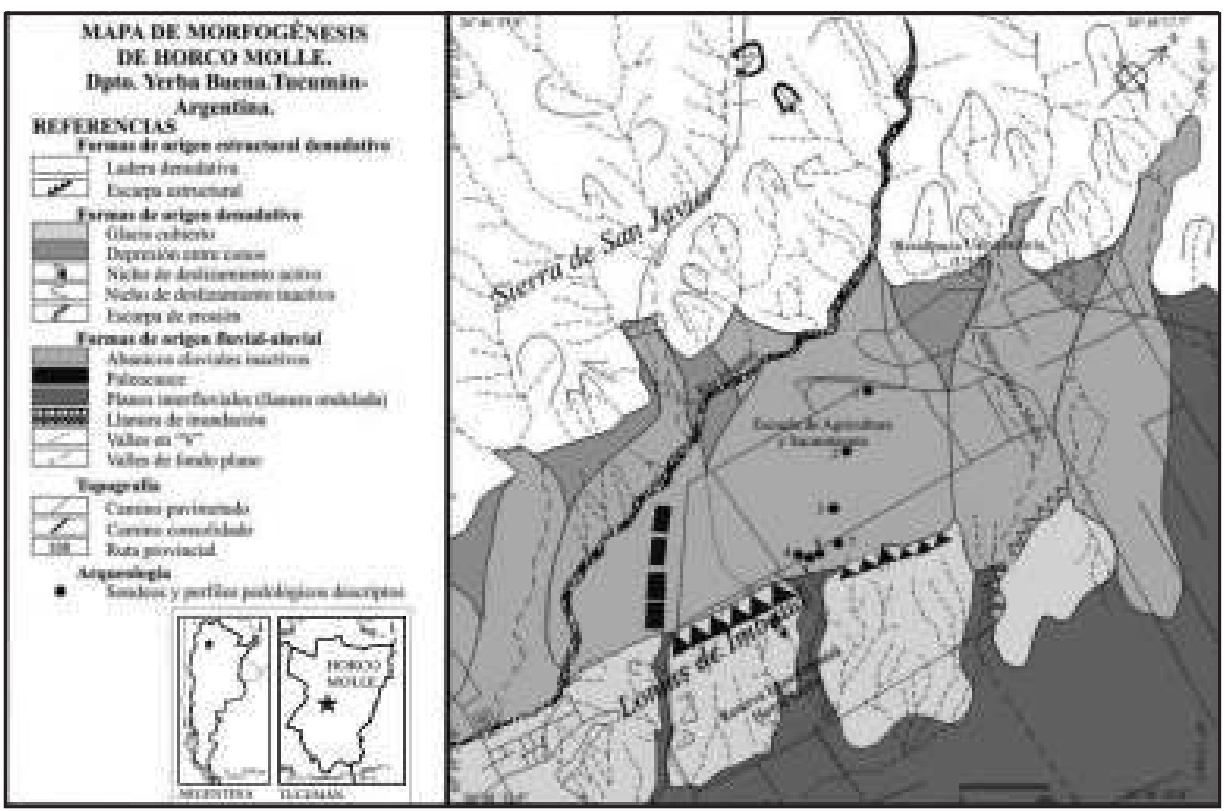

Figura 2. Mapa morfogenético de Horco Molle y distribución de sondeos. (Maldonado et al. 2009).

\section{Resultados}

Desde el punto de vista geomorfológico se determino la presencia de formas de origen estructural-denudativo (ladera denudativa, escarpa estructural), de origen denudativo (glacis cubierto, nichos de deslizamiento activos e inactivos, escarpa de erosión) y de origen fluvialaluvial (abanicos aluviales, planos interfluviales, cauce principal, paleocauce, valles en "V" y de fondo plano) (figura 2).

Pedoestratigráficamente, en el abanico aluvial del río Muerto se distinguió un paleosuelo loéssico al que se superponen capas de fanglomerados y el suelo actual (figura 3). El paleosuelo fue detectado en el sector medio y distal (perfiles 3, 5, 6 y 7). En el sector medio fue erosionado quedando remanentes (perfil 2) o sin el horizonte A (perfil 3), por factores naturales y/o antrópicos, aunque no son suficientes los datos para evaluarlo. Posee un espesor mayor a 50 $\mathrm{cm}$, un horizonte $2 \mathrm{~A}$ con estructura de prismas que rompen a prismas menores y textura franco limosa, y un horizonte $2 \mathrm{~B}$ (o $3 \mathrm{~B}$ ) con estructura de prismas que rompen a prismas menores, textura franco arcillosa y cutanes continuos recubriendo paredes y poros.

De los sectores sondeados, sólo 2 ofrecieron contextos claramente definidos, el sondeo 3 y el sector 7 , los cuales se desarrollan a continuación.

El sondeo 3 (ver figura 2) presentó un perfil pedológico con horizontes $\mathrm{A} / \mathrm{C} / 2 \mathrm{C} / 3 \mathrm{~B} /$ $3 \mathrm{BC}$. Sobre el paleosuelo $(3 \mathrm{~B} / 3 \mathrm{BC})$ se distinguió una superficie de ocupación con rubefacción 


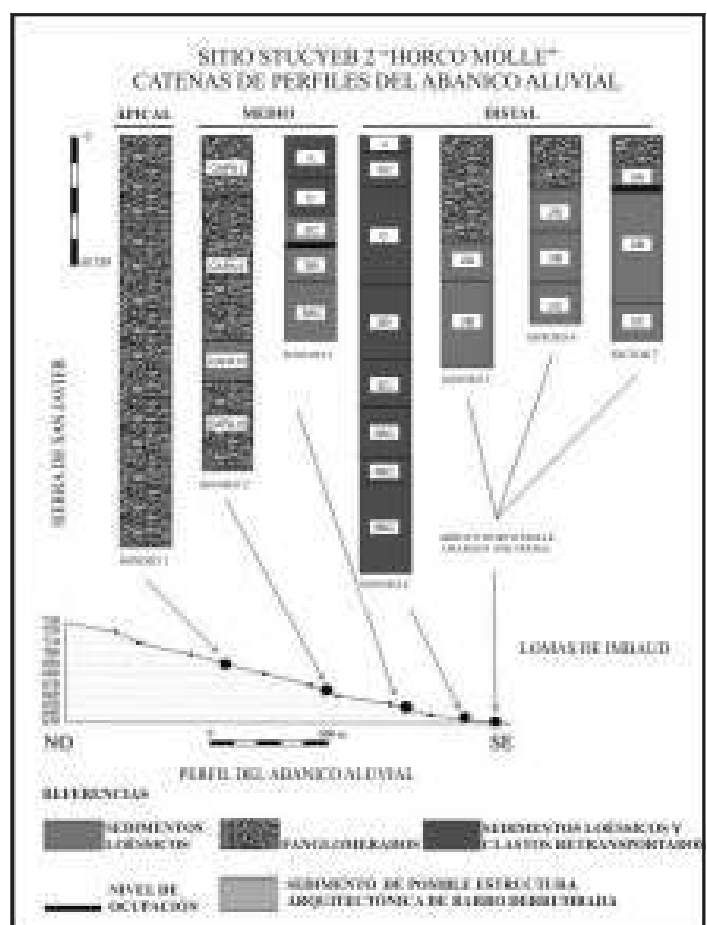

Figura 3. Perfiles pedoestratigráficos del abanico aluvial del río Muerto (Maldonado et al. 2009).

y se encontraron fragmentos cerámicos fracturados in situ de parte de una vasija acanalada, fragmentos de otras vasijas y trozos de carbón. Se trataría de un locus de actividades domésticas (figura 4). En el horizonte 2C se evidenció dispersión de materiales cerámicos y fragmentos de un molino. También se observaron dos manchas oscuras alargadas, posiblemente generadas por maderas descompuestas in situ (figura 4), que asociadas a las diferencias pedológicas de este horizonte respecto a los restantes (color más claro, mayor compactación) permiten inferir que una estructura perecedera se habría desplomado sobre el piso habitacional aplastando la vasija acanalada y el molino de arenisca dejados como residuos de facto (sensu Schiffer 1987).

El sector 7 (ver figura 2) presentó un perfil pedológico de capa detrítica/2A/2B/2C. En el horizonte 2A se distinguió una concentración de artefactos (cuadrículas H2, I2) del que proceden fragmentos cerámicos de una misma vasija, 2 puntas de proyectil apedunculadas y fragmentos del ápice de una tercera, 1 FNRC, 1 artefacto de formatización sumaria, 1 artefacto burilante + FNRC, 1 raclette + muesca + perforador, 1 núcleo y 4 lascas, asociados a una lente de arcilla, que serían depósitos de origen cultural. En algún momento se enterró una urna funeraria por debajo del nivel cultural, en el horizonte 2B, que contenía tres cuentas líticas, una vasija zooantropomorfa y restos óseos de 3 infantes (Colaneri et al. 2003) (figura 5) que consistiría en un entierro secundario (Maldonado et al. 2009).

Con relación al análisis cerámico (total: 2287 fragmentos), los grupos de pastas identificados macroscópicamente fueron 4 : 


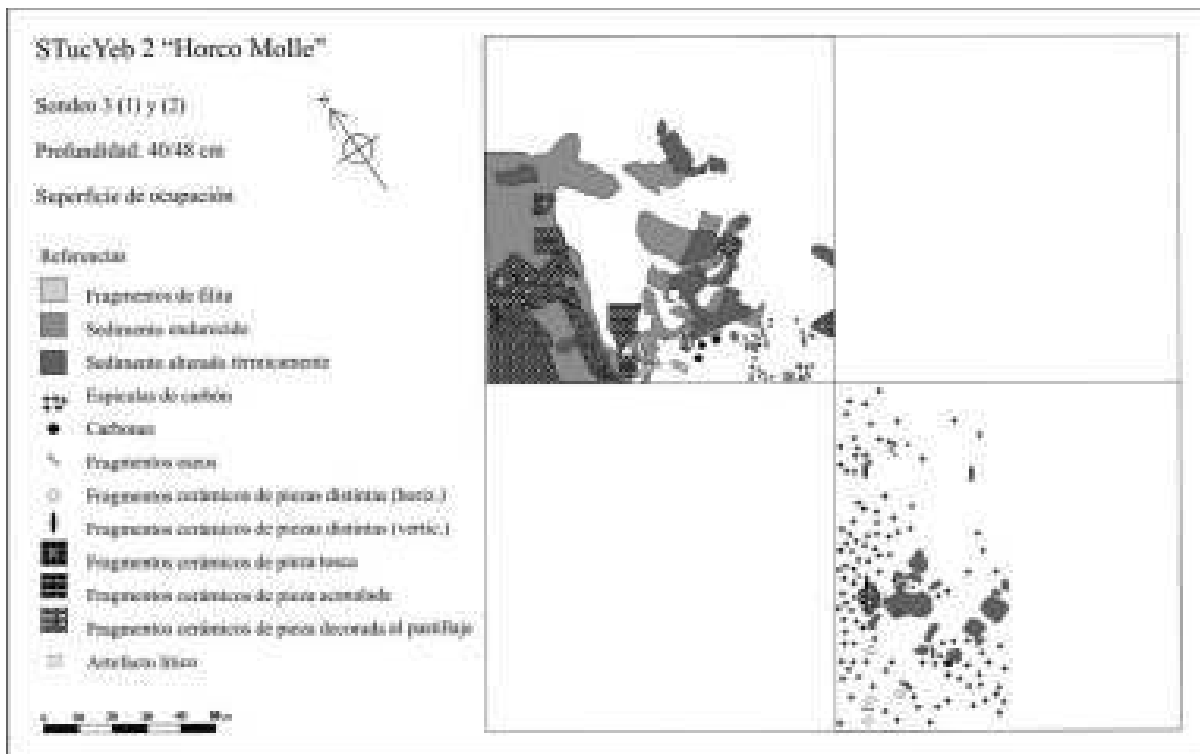

\section{STucYeb 2 "Horco Molle"}

Sanden 3 (1) 9 (a)

Fofandatat $200 \mathrm{in}$

Capas:

Renowian

(7) Fevonin is fibe

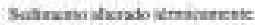

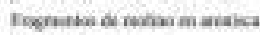

4. Lequalas de arties

- Conear

\& fegresta osere

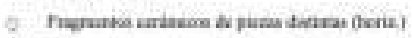

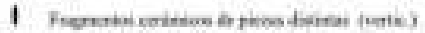

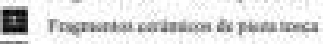

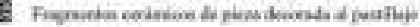

Anefason itroo

Tras \& estem decerpestes
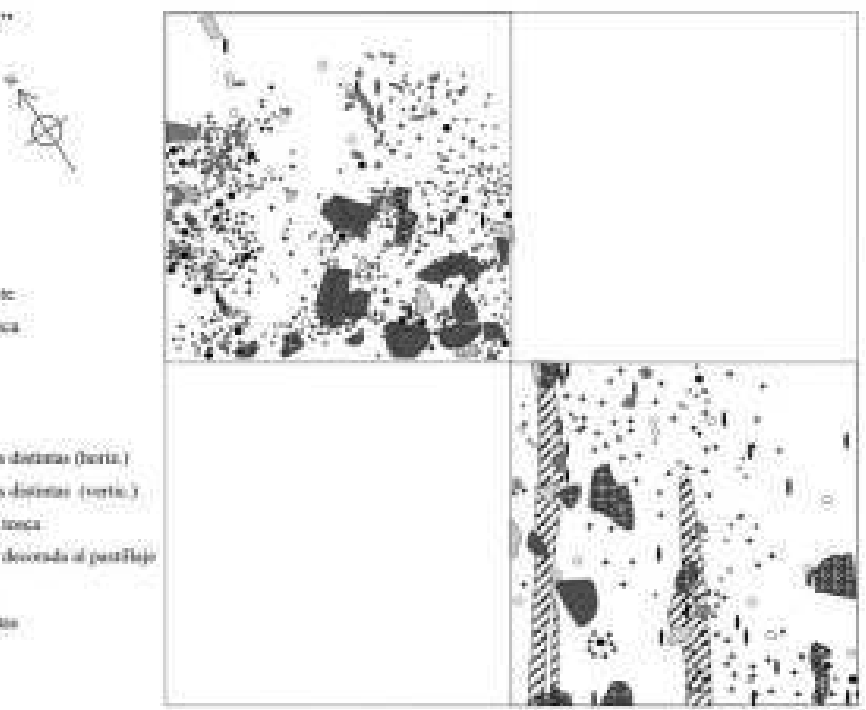

Figura 4. Sondeo 3, superficie de ocupación (arriba) y relleno superpuesto a la misma (abajo) (Maldonado et al. 2009).

A-inclusiones de filitas predominantes, muscovita y cuarzo, tamaño no uniforme, distribución regular (filita y muscovita), e irregular (cuarzo), densos (filita) y poco densos (muscovita y cuarzo), textura laminar o porosa, fractura irregular, color del núcleo de naranja fuerte a gris oscuro y negro;

$B$ - inclusiones de muscovita y granitos de cuarzo, tamaño no uniforme, distribución regular (mica) e irregular (cuarzos), poco densos, textura compacta, fractura regular, color del núcleo de gris claro a naranja; 
C- inclusiones predominante de muscovita (tal vez inclusión intencional) y granos de cuarzo, tamaño no uniforme, distribución regular (muscovita), irregular (cuarzos), densos (muscovita) y poco densos (cuarzo), textura laminar, fractura regular, núcleos variantes de negro a naranja claro;

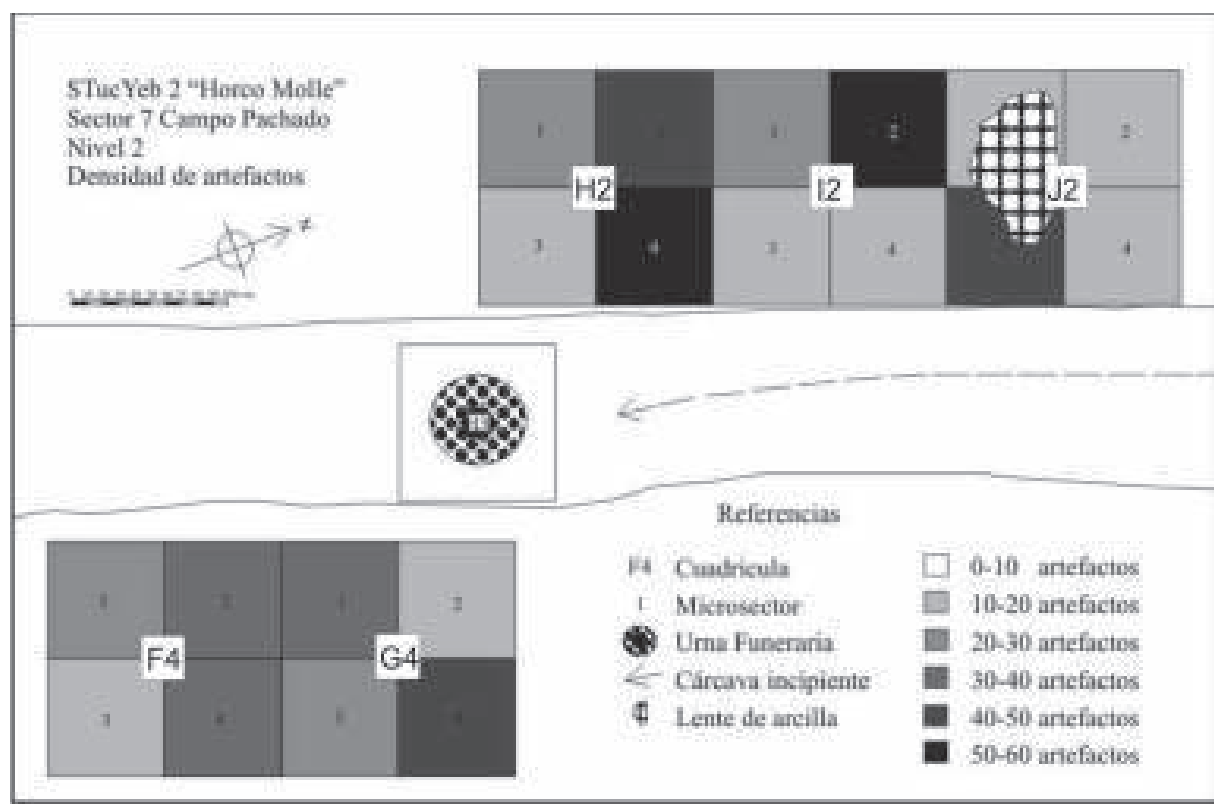

Figura 5. Sector 7, densidad de hallazgos (Maldonado et al. 2009).

$D$ - inclusiones de muscovita, tamaño uniforme, distribución regular, poco densa o densa, textura compacta, fractura regular, color del núcleo entre naranja y gris oscuro. Hipotéticamente, las materias primas de las inclusiones serían locales, obtenidas de la molienda de filita y granito.

Cuantitativamente, el $94,14 \%$ son fragmentos de pasta del grupo A, el 1,22\% del grupo B, el 2,88\% del grupo C, y el 1,74\% del grupo D. El 98,33\% son no decorados y el 1,66\% son decorados. Entre los acabados de superficie predominan los fragmentos alisados $(80,34 \%)$ sobre toscos $(2,17 \%)$, pulidos (1,02\%) y engobados $(0,48 \%)$, y entre las técnicas decorativas prevalecen fragmentos pintados $(36,84 \%)$, sobre incisos $(23,68 \%)$, incisos y modelados $(15,78 \%)$, acanalados $(5,26 \%)$, grabados $(5,26 \%)$ y estampados $(2,63 \%)$. Se establecieron 15 tipos cerámicos, 6 no decorados definidos aquí y 9 decorados definidos por Heredia (1975: 121-131), predominando entre los no decorados el Horco Molle alisado $(75,55 \%)$, seguido por los demás tipos en frecuencias inferiores al 3\%, y dominando entre los decorados el Candelaria rojo pintado $(0,48 \%)$ sobre el Candelaria gris inciso $(0,29 \%)$, Candelaria modelado $(0,26 \%)$ y sobre otros. Los fragmentos inclasificados son en su mayor parte erodados (figura 6).

Los tipos decorados identificados se hallan particularmente presentes en las fases Chuscha y Choromoro (Heredia 1975) (tabla 1). Comparando los "rasgos diagnósticos" de ambas fases y su análisis para Horco Molle (tabla 2), los de Horco Molle presentan mayores similitudes con los de la Fase Chuscha $(1,2,4,5,7,8)$ que con los de la Fase Choromoro (3 y 6 ). 
El análisis estratigráfico de los porcentajes de tratamientos de superficie y técnicas decorativas para el sondeo 4 (1,40 m prof.) (ver figura 2), muestra cambios que podrían indicar tendencias temporales. No obstante los fragmentos cerámicos presentan gradualmente mayor alteración por arrastre hídrico en sentido estratigráfico ascendente (reducción de tamaño, redondeo de formas y abrasión de bordes) perdiendo el acabado de superficie, haciendo descender los porcentajes de toscos, pulidos y engobados y aumentar los de inclasificados y de alisados, manifestándose como husos que simulan cambios cronológicoestilísticos (figura 7).

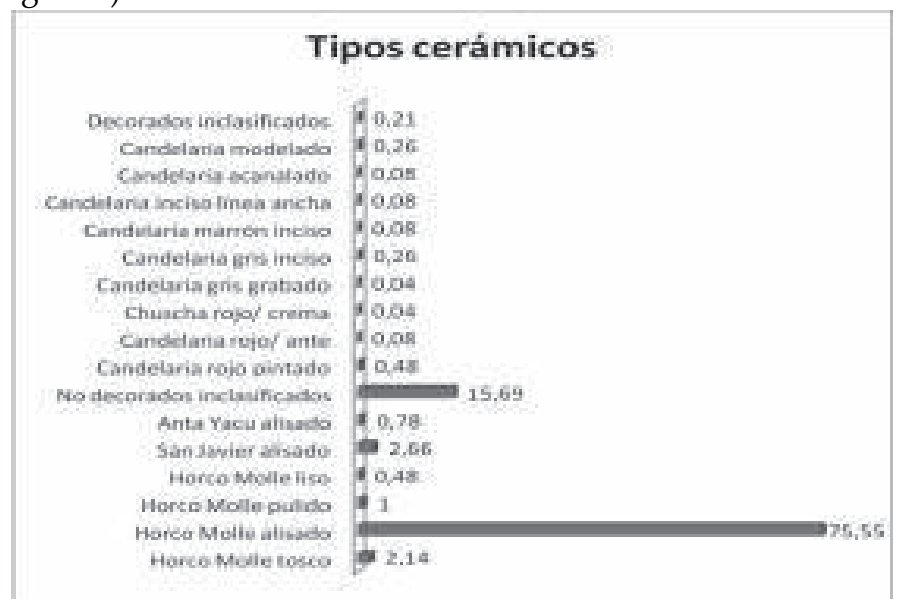

Figura 6. Porcentajes de tipos cerámicos.

\begin{tabular}{|c|c|c|c|c|c|c|}
\hline \multicolumn{7}{|c|}{ STucYeb 2 "Hordo Male" } \\
\hline \multirow{2}{*}{$\begin{array}{c}\text { Tipces decoradces } \\
\text { STucYet } 2\end{array}$} & \multicolumn{6}{|c|}{ Fases Candelaria } \\
\hline & Chuschs & Charemoro & Molleyaco & Pugactico & Quebractisl & 519. Bartbers \\
\hline Candelaria roje pintado & $x$ & $x$ & & & & \\
\hline Candelaria rojaiante & $x$ & $x$ & $x$ & & & \\
\hline Chuscha rojokrems & $x$ & & & & & \\
\hline Candelaria gris grabado & $x$ & $x$ & $x$ & $x$ & $x$ & $x$ \\
\hline Candelaria gris inclao & $x$ & $x$ & $x$ & & $x$ & \\
\hline Candelaria marrón inciso & $x$ & $x$ & $x$ & & $\mathrm{x}$ & \\
\hline Candelaria inciso linea & $x$ & $x$ & & & & $x$ \\
\hline Candelaria acanalodo & $x$ & $x$ & $x$ & $x$ & & \\
\hline Candelaria modelado & $x$ & $x$ & & & & \\
\hline
\end{tabular}

Tabla 1. Tipos cerámicos decorados identificados y su presencia/ausencia en las fases de Candelaria. 


\begin{tabular}{|c|c|c|}
\hline \multicolumn{3}{|c|}{ Rasgos cerimicas diagnéstivos } \\
\hline Fasechischa & Fase Chromoev & STucYeb 2 Tlborco Molle" \\
\hline Tamano do moesta: 3862 fragmentas. & $\begin{array}{l}\text { Tamino de misests } 3718 \\
\text { fragmentos. }\end{array}$ & $\begin{array}{l}\text { Tama în de moksta: } 22 \times 7 \\
\text { fogmantos }\end{array}$ \\
\hline $\begin{array}{l}\text { 1. Cerrimica decuenda }(3,75 \%) \text {. } \\
\text { Cerainaica so decomada }(96,25 \%) \text {. }\end{array}$ & $\begin{array}{l}\text { 1. Ceramica decirnade }(4,49 \%) \\
\text { Cerimica no decorada }(95.51 \%) \text {. }\end{array}$ & $\begin{array}{l}\text { 1. Ceramien deccradi }(1,66 \%) \text {. } \\
\text { Ceraimica no deceraib }(98,13 \%) \text {. }\end{array}$ \\
\hline $\begin{array}{l}\text { 2. Cerimica grabada }(27.18 \%) \text {. } \\
\text { Ceraimiea ineisa }(72,82)\end{array}$ & $\begin{array}{l}\text { 2. Ceramien gribada }(30.54 \%) \text {, } \\
\text { Ceraimica inciea }(\omega), 46 \%) \text {. }\end{array}$ & $\begin{array}{l}\text { 2. Ceramica zrabada }(38,18 \%) \text {. } \\
\text { Cerimica incise }(81.81 \%) \text {. }\end{array}$ \\
\hline $\begin{array}{l}\text { 3. Decrandos }(30.28 \%) \\
\text { Lisas }(69.72 \% 6)\end{array}$ & $\begin{array}{l}\text { 3. Decorados }(40,58 \%) \text {. } \\
\text { Lisos }(59,42 \%) \text {. }\end{array}$ & $\begin{array}{l}\text { 3. Deconados }(12,77 \% \text { h } \\
\text { Lisos }(47,22 \%) \text {. }\end{array}$ \\
\hline 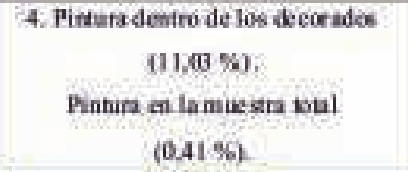 & 4 sibdikos. & $\begin{array}{l}\text { 4. Phiuri dentro de los decotudes } \\
\qquad(36,84 \%) \text {. } \\
\text { Pinain es la muestra totat }(0,61 \%) \text {. }\end{array}$ \\
\hline 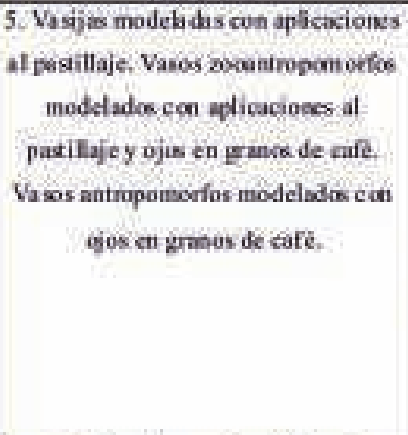 & 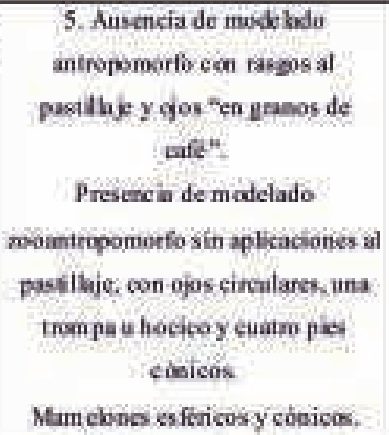 & 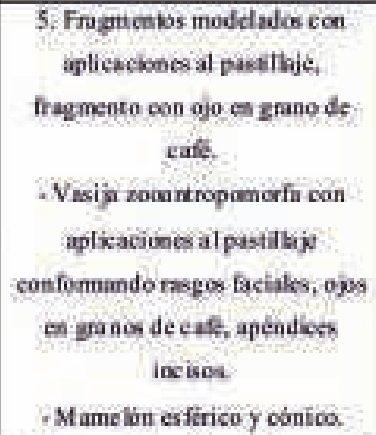 \\
\hline 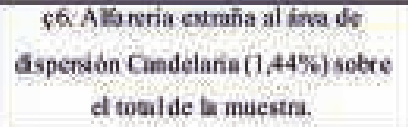 & 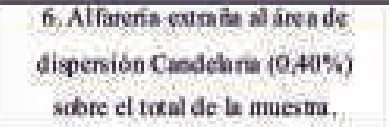 & 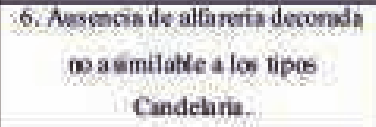 \\
\hline 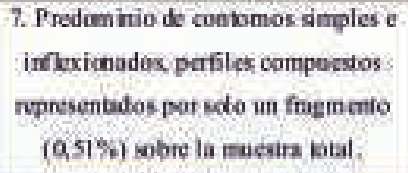 & $\begin{array}{l}\text { T. Aumerifo de los perfiles } \\
\text { compuesos }(4,08 \%) \text {. }\end{array}$ & $\begin{array}{c}\text { 7. Un sole fragmento con punto } \\
\text { angular }(0,04 \%) \text {. }\end{array}$ \\
\hline 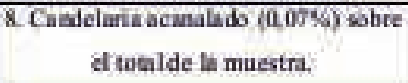 & $\begin{array}{l}\text { 8. Cabdelaria touna lado }(0,35 \%) \\
\text { sobre el tokal de la muestri }\end{array}$ & $\begin{array}{l}\text { 8. Candelaria ac analado }(0,08 \%) \\
\text { sobre el todalde at muestri. }\end{array}$ \\
\hline
\end{tabular}

Tabla 2." Rasgos diagnósticos" de las fases Chuscha y Choromoro (Heredia 1975) y su análisis para Horco Molle.

Por otro lado, en el conjunto lítico recuperado (total: 65 artefactos), la materia prima predominante es el cuarzo (57), seguido por cuarcita (2), ś́lice (1), calcedonia (1), vulcanita indiferenciada (1), e indiferenciadas (3).

Los desechos de talla (55) se componen de lascas enteras (7,22\%), lascas fracturadas con talón $(45,45 \%)$, lascas fracturadas sin talón $(32,72 \%)$ y desechos indiferenciados $(14,54 \%)$. 


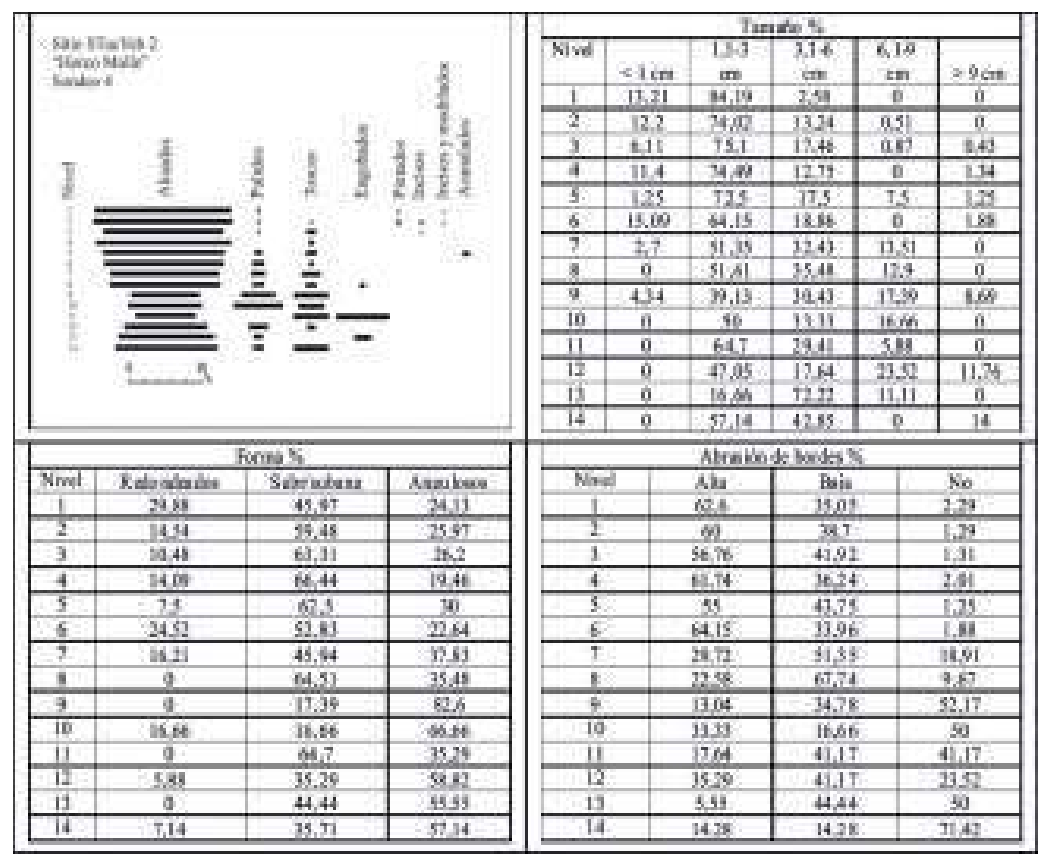

Figura 7. Sondeo 4. Distribución estratigráfica de frecuencias de acabados de superficies y técnicas decorativas, junto a trazas de alteración.

El Índice de Fragmentación es de 0,86 y el Número Mínimo de Desechos de 29, sobre los que se realizaron los análisis siguientes. Predominan lascas de 15 a 19,9 mm de longitud (9), de 10 a $14,9 \mathrm{~mm}$ de ancho (11), y de 0 a $4,9 \mathrm{~mm}$ de espesor (14). El origen de las extracciones señala la presencia exclusiva de lascas internas (lascas angulares: 11). Predominan talones de talla/retalla (> a 7mm) (26) sobre los de retoque (7-2,1 $\mathrm{mm}$ ) (3) y de microretoques (<a 2 $\mathrm{mm}$ ) (0). Domina la frecuencia de talones lisos (15), sobre los lisos naturales (7), filiformes (2), naturales (1), diedros (1) y facetados (1). La frecuencia de talones corticales (11) es menor respecto al resto (18), y hay corteza en la cara dorsal de algunos desechos (5 sobre un total de 29). También se halló 1 núcleo en cuarzo con corteza en la plataforma de percusión.

El conjunto de artefactos formatizados consiste de 8 piezas (6 enteras y 2 fracturadas) y hay sólo 2 artefactos con rastros complementarios. Los grupos tipológicos identificados son: punta de proyectil apedunculada en cuarzo ( 3 enteras, 2 fragmentos de ápice), triangulares de base escotada sobre lascas no diferenciadas o formas bases no diferenciadas, confeccionadas mediante micro retoque marginal, retoque marginal, retoque parcialmente extendido y retoque extendido; raclette + muesca + perforador en sílice (1) sobre lasca no diferenciada, confeccionado mediante microretoque marginal (raclette), retoque marginal (perforador), lascado simple de formatización y microretoque marginal (muesca); artefacto de formatización sumaria en calcedonia (1) sobre lasca angular, confeccionado mediante microretoque marginal; artefacto burilante + FNRC en vulcanita indiferenciada (1) sobre lasca angular, confeccionado mediante lascado simple de formatización; y FNRC en cuarzo (1) sobre lasca angular. 
La abundancia del cuarzo respecto a las demás materias primas indica una fuente de aprovisionamiento local. La presencia de corteza en un núcleo, cara dorsal y talones de algunos desechos de talla sugiere una fuente de aprovisionamiento secundaria (por cercanía el lecho del río Muerto y del arroyo Anta Yacu). El cuarzo recolectado habría sido trasladado al asentamiento donde se extrajeron formas bases no estandarizadas mediante talla por percusión y se formatizaron las puntas de proyectil mediante retoque y microretoque a presión, marginales y parcialmente entendidos. El sílice, la vulcanita y la calcedonia serían foráneas dada la escasa cantidad en el conjunto, y se emplearon para elaborar artefactos de filo.

\section{Discusión}

Los hallazgos de contextos de ocupación del sondeo 3 y del sector 7 , más otro conocido como sector II (Cano 2003), evidencian ocupaciones en lugares bajos del piedemonte (en el abanico aluvial), además de haberlas en espacios elevados como en el sector VII (Míguez $2005)^{1}$ (figura 8). Ello no apoya la propuesta de un uso diferencial de los sectores altos de glacis cubierto (doméstico) respecto a los sectores bajos del piedemonte (funerario) (Míguez 2005). Hipotéticamente, la dinámica geomorfológica del piedemonte afectó diferencialmente la visibilidad arqueológica, siendo menor en los sectores bajos respecto a los elevados, generando distorsión en la percepción del patrón de asentamiento con una mayor representación de ocupaciones arqueológicas en lomadas, tal como se ha percibido para Horco Molle y para otros sitios con cerámica Candelaria (Maldonado et al. 2009).

Las ocupaciones formativas documentadas en este y otros trabajos (Cano 2003, Míguez 2005) se asientan sobre un paleosuelo loéssico con horizontes iluviales bien diferenciados. En el valle de Tafí y la cuenca de Tapia-Trancas se documentaron también asentamientos formativos sobre paleosuelos pedológicamente desarrollados, que junto con otros proxies sugieren condiciones paleoclimáticas húmedas (Sampietro et al. 2003).

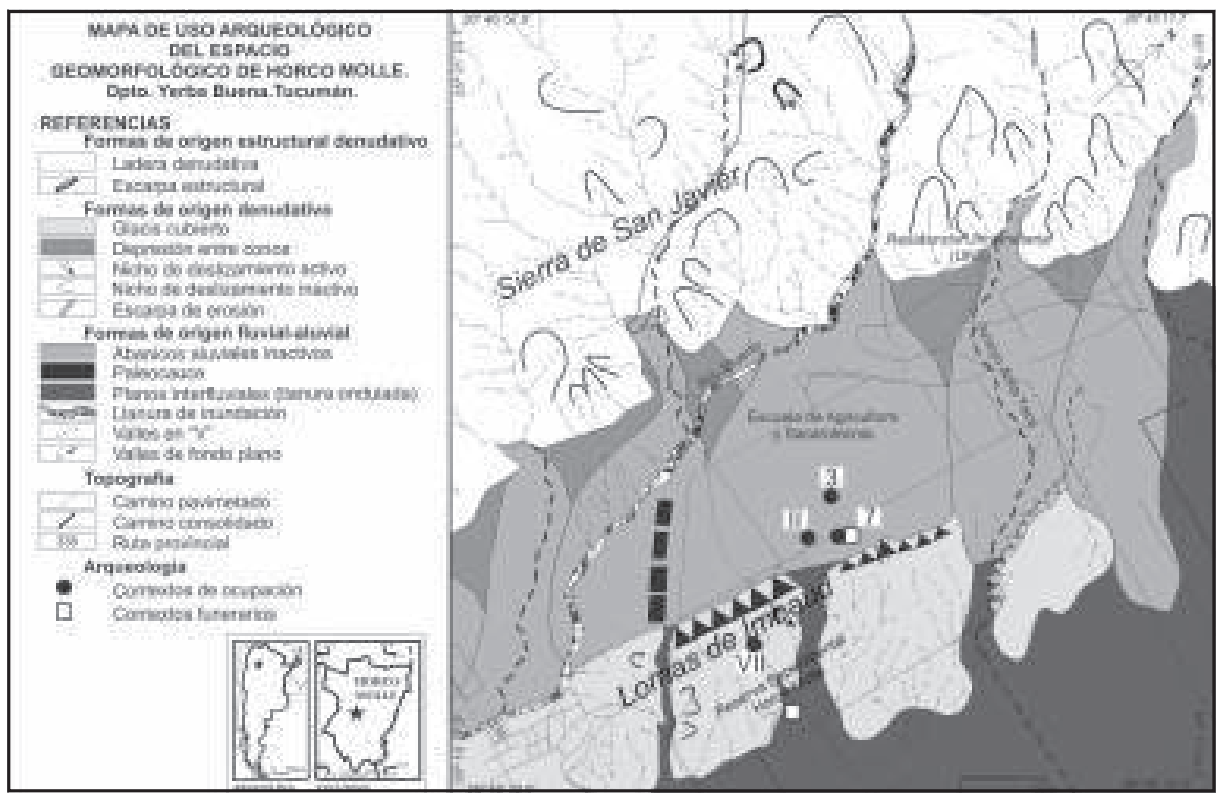

Figura 8. Mapa de uso del espacio geomorfológico. 


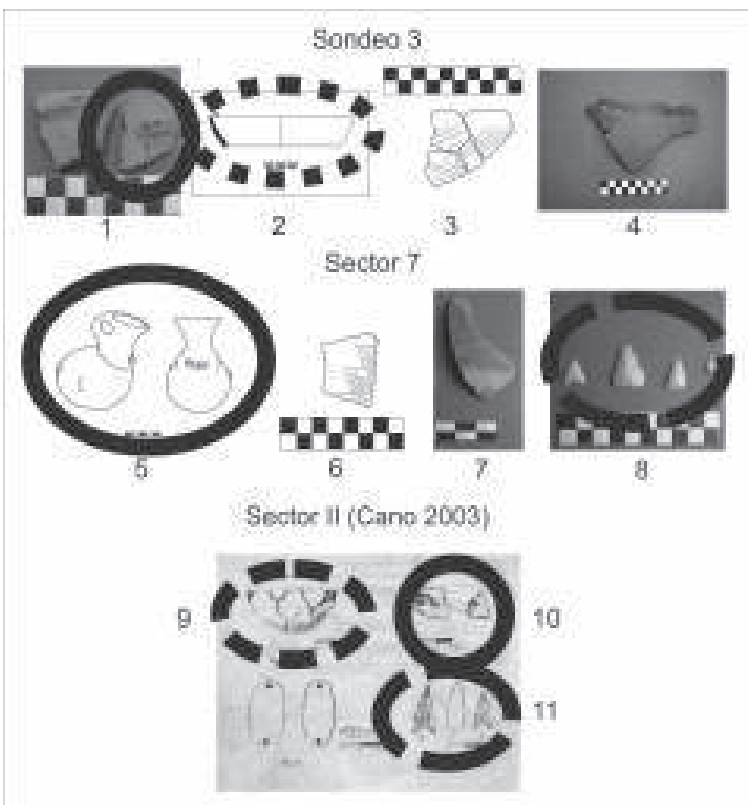

Figura 9. Comparación de artefactos cronológicamente diagnósticos de contextos de ocupación

Algunos datos a nivel artefactual permiten inferir la modalidad de las relaciones de estos grupos humanos formativos con la geología local.

Las vasijas en general tenían bajo costo de producción considerando que: (a) las materias primas de las inclusiones (pizarra y filita) para el grupo de pasta dominante (grupo A) son abundantes en el medio local al igual que las arcillas (Mon y Suayter 1972-1974); (b) el tamaño grueso a muy grueso de las inclusiones de pastas del grupo A, no uniformes, que podría implicar escaso trabajo en la molienda; (c) la escasa inversión de trabajo en decoración (1,66\%); (d) el predominio del alisado (80,34\%) sobre pulido o engobado; (e) los colores variables de núcleos y superficies, que indicarían escaso control del ambiente de cochura, acorde con cocción a cielo abierto (Rye 1981); (f) la morfología reconstruida o registrada de las vasijas del tipo más popular (no restringidas y restringidas independientes) (Horco Molle alisado: 75,55\%), que es acorde con usos domésticos y funerarios, indicando una producción para satisfacer necesidades inmediatas. El bajo costo de obtención de la materia prima pudo influir en la baja inversión de trabajo en la manufactura y en la ausencia de reuso y reclamación de cerámica.

Coincidimos con Míguez y Gramajo Bühler (2007) en que la variedad de materias primas más frecuente entre los artefactos líticos es el cuarzo (que sería local), hallándose en proporciones ínfimas la arenisca (Míguez y Gramajo Bühler 2007), filita (Cano 2003), cuarcita, sílice, calcedonia y vulcanita, siendo las tres últimas posiblemente foráneas ${ }^{2}$. En cuarzo se elaboraron puntas de proyectil, y en las restantes materias primas se realizaron artefactos de filo natural y/o formatizado dada su mayor aptitud que la del cuarzo para tal fin. El cuarzo, procedente de una fuente secundaria (lecho de un río), presenta múltiples fisuras que debieron condicionar la obtención de formas bases con bordes adecuados para elaborar artefactos de 
filo, evidenciado en el Índice de Fragmentación de los desechos de talla $(0,86)$. La ausencia de reuso o reciclaje en puntas de proyectil se relacionaría con la baja inversión de trabajo en la obtención del cuarzo. Las materias primas para elaborar artefactos de filo implicarían mayor costo de obtención, reflejado en el reciclaje del instrumento en sílice.

Costos de producción y usos también debieron influir en los procesos de depositación. El entierro en urna del sector 7 contenía tres cuentas de collar (en anfíbol, roca volcánica y crisocola, todas foráneas) y una vasija pequeña zooantropomorfa decorada por incisión, modelado y pastillaje (Colaneri et al. 2003, Ruiz 2003). En el conjunto cerámico total la decoración es del 1,66\% y dentro de ésta la combinación de incisión, modelado y pastillaje es del $15,78 \%$, por lo que el trabajo en decoración no es común. Vasijas de este tipo se hallan en contextos funerarios y no en pisos de ocupación (Ryden 1936, Heredia 1975). En nuestro conjunto lítico y en otros ya analizados de Horco Molle (Cano 2003, Míguez y Gramajo Bühler 2007) no se hallaron artefactos en las materias primas de las cuentas, ni cuentas indudables en otras materias primas. La inclusión de estas cuentas como ajuar respondería a su alto costo de producción, a sus características ornamentales y a un uso comúnmente funerario (Heredia 1975). La depositación como desechos de facto de la vasija acanalada (pasta grupo A) y el molino (arenisca), de la ocupación del sondeo 3, respondería a que están hechos en materias primas locales, además de sus tamaños, pesos y grado de desgaste (Joyce y Johannessen 1996).

Respecto a la cronología relativa del sitio Horco Molle, algunos artefactos recuperados de los contextos de ocupación excavados por nosotros y por otro autor (Cano 2003) ofrecen elementos de juicio.

En la figura 9 se observa que dichos contextos comparten elementos tales como el tipo Candelaria modelado con ojos "en granos de café" $(1,5,10)$, fragmentos de pucos de contorno simple $(2,9)$, además de fragmentos Candelaria monocromo rojo y puntas de proyectil triangulares de base escotada en cuarzo $(8,11)$. Se agrega la presencia de Candelaria gris grabado (3), Candelaria gris inciso (6) y Candelaria acanalado (4). Estos tipos cerámicos juntos, y puntualmente el Candelaria modelado y Chuscha rojo/crema, son asignables a la fase Chuscha (200-400 DC) (Heredia 1975).

El análisis del conjunto cerámico total también sugiere que los grupos formativos arribaron a Horco Molle durante la Fase Chuscha (200-400 DC), aunque hay similitudes con algunos aspectos cerámicos de la Fase Choromoro (400-700 DC) y no se descarta la presencia humana durante ésta última. Se documentó cerámica Hualfín pintado y un fragmento fino negro pulido con reticulados incisos (Míguez 2005) que llevarían la cronología a las Fases Molleyaco (400-700 DC) y Rupachico (700-1000 DC). Los "rasgos diagnósticos" cuantitativos de Heredia (1975) para diferenciar entre las fases Chuscha y Choromoro presentan diferencias comparativas muy estrechas (mayormente de $4 \%$ o menores, 120 tiestos sobre muestras de 3000 fragmentos), por lo que es dudosa su utilidad metodológica para diferenciar entre ambas fases claramente, como en nuestro caso de estudio ${ }^{3}$.

La cronología relativa propuesta para Horco Molle es comparable a la sugerida para el sitio Bajada de la Ovejería (valle de La Sala), ubicado al oeste de la Sa. de San Javier, a 4 Km de Horco Molle, donde se hallaron fragmentos cerámicos con ojos en granos de café al pastillaje y lágrimas incisas y un fragmento Condorhuasi polícromo. El cuarzo es la materia prima 
lítica más común, pero se registraron traquitas y obsidiana perlítica foráneas (Angiorama et al. 1992). También se conocen puntas de proyectil triangulares de base escotada en cuarzo y obsidiana, de colecciones locales.

Fuera de Selvas Occidentales meridionales, se encontraron conjuntos artefactuales similares en contextos domésticos del valle de La Ciénega (Dpto. de Tafí del Valle), con dos niveles de ocupación asignados a Candelaria con cerámica monocroma roja, lascas, núcleos y puntas de proyectil triangulares de base escotada (Bernasconi de García y Baraza de Fonts 1985). Cremonte (1996) asigna la cultura material de La Ciénega a la Tradición Tafí (fechados de $1970 \pm 120$ años AP o 31-54 años cal DC (AC-0580) carbón vegetal; y $1240 \pm 80$ años AP o 778-797 años cal DC (AC-0721) carbón vegetal), pero destaca relaciones cerámicas notorias con la Fase Chuscha y también con la Fase Choromoro, y la presencia de artefactos de filo sobre lascas, dos puntas de proyectil triangulares de base escotada en cuarzo y una triangular con pedúnculo en obsidiana. En el sur de valle de Santa María se excavaron dos recintos hallándose cerámica Vaquerías y fragmentos pulidos semejantes a los de estilo Candelaria (modelados al pastillaje de rasgos zoomorfos, cejas punteadas, ojos en granos de café), puntas de flecha triangulares de base escotada en obsidiana (fechado de $1940 \pm 80$ años AP o 103 años cal AC-310 cal DC (LP-1541) sobre carbón vegetal) (Palamarczuk et al. 2006). En el valle del Cajón se excavó un piso de ocupación con cerámica monocroma roja, modelados zoomorfos incisos similares a los de Candelaria, cerámicas pulidas, fragmentos Vaquerías entre otros, pero aquí con puntas de proyectil pedunculadas en obsidiana, basalto y roca volcánica gris no identificada (fechado de $1878 \pm 57$ años AP o 70-220 años cal. AD (AA 67778) en carbón) (Scattolin et al. 2007). La asociación observada de cerámica al pastillaje con cejas y ojos en granos de café, modelados zoomorfos, cerámica monocroma roja, y puntas de proyectil triangulares de base escotada se da entre Selvas Occidentales meridionales y al menos los valles de Tafí y Santa María, y casi desde el comienzo de la Era Cristiana. Consideramos que en Horco Molle las ocupaciones comenzaron en la Fase Chuscha (200$400 \mathrm{DC})$, o posiblemente antes.

\section{Conclusiones}

Condiciones paleoclimáticas húmedas y suelos loéssicos desarrollados favorecieron el asentamiento de grupos formativos con cerámica Candelaria sobre unidades geomorfológicas diferentes en el piedemonte (abanico aluvial y glacis cubierto) durante las Fases Chuscha (200-400 DC) y Choromoro (400-700 DC), y posiblemente Molleyaco (400-700 DC) y Rupachico (700-1000 DC). La disponibilidad y adecuación general de ciertas rocas y minerales para la producción de artefactos líticos y cerámicos influyeron en bajos costos de obtención (con algunas excepciones), de manufactura, en los procesos de depositación (de residuos de facto, ajuar funerario y desechos secundarios), escaso reuso (reciclaje) y ausencia de reclamación de artefactos.

Los resultados son de interés para la arqueología de Selvas Occidentales meridionales, dadas las observaciones efectuadas respecto a la dudosa efectividad de los "rasgos cerámicos diagnósticos" para establecer diferencias temporales entre las fases Chuscha y Choromoro. Destacamos el conocimiento de la producción lítica, casi desconocida para las sociedades con cerámica Candelaria en general, y para las fases Chuscha y Choromoro puntualmente. Se destaca la importancia de considerar la influencia de los procesos de arrastre hídrico en la seriación cerámica dado que sus efectos pueden simular husos de cambios cronológicos- 
culturales, considerando que restan por resolverse problemas cronológicos y que estos procesos son generalizados en Selvas Occidentales. Los sesgos generados por el arrastre hídrico también deben considerarse en colecciones de tiestos superficiales. Finalmente, la mayor representación de asentamientos en lugares elevados para sitios con cerámica Candelaria resultaría, en muchos casos, producto de una percepción distorsionada del patrón de asentamiento influida por la dinámica geomorfología del piedemonte sobre la visibilidad arqueológica.

\section{Agradecimientos}

Este trabajo fue solventado por la Secretaría de Ciencia y Técnicas (UNT) proyecto CIUNT 26/G440 y la ANPCyT proyecto PICT 31680.

\section{Notas}

${ }^{1}$ Se utilizan las designaciones de sectores de los autores mencionados.

${ }^{2}$ Las materias primas de tres cuentas halladas en el entierro del sector 7 (anfíbol, roca volcánica y crisocola) también serían foráneas (Ruíz 2003).

${ }^{3}$ No cuestionamos el sustento empírico de dichas fases (una superposición estratigráfica y recolecciones de superficie). Sólo dudamos de su utilidad para diferenciarlas claramente, aunque también depende de la resolución cronológica de los conjuntos cerámicos analizados.

\section{Bibliografía citada}

Angiorama C. I., M. A. Caria, L. A. Moya y C. Taboada. 1992. Bajada de La Ovejería: un sitio Candelaria. II Congreso Nacional de Estudiantes de Arqueología: 1-6. Rosario.

Aschero, C.

1975. Ensayo para una clasificación morfológica de artefactos líticos aplicada a estudios tipológicos comparativos. Informe al CONICET. Bs As.

Aschero, C.

1983. Ensayo para una clasificación morfológica de artefactos líticos aplicada a estudios tipológicos comparativos. Apéndices A-C. Revisión. Cát.de Ergología y Tecnología. (FFyLUBA). Bs As.

Bernasconi de García, M. T. y A. N. Baraza de Fonts.

1985. Estudio arqueológico del Valle de la Ciénaga (Departamento Tafí, Prov. de Tucumán). Anales de Arqueología y Etnología 36-37: 117-138.

Butzer, K. W.

1989. Arqueología- una ecología del hombre: método y teoría para un enfoque contextual. Ed. Bellaterra, Barcelona.

Cano, S. F.

2003. Aproximación al estudio de los procesos de formación de sitios en el área pedemontana de la Sierra de San Javier: análisis preliminar del sitio STucYeb 2 (Depto. Yerba Buena, Tucumán). Universidad Nacional de Tucumán. Inédito.

Colaneri, M. G., A. Calisaya, A. Gerónimo, E. Milena, A. González, V. Vargas, S. Rodríguez, M. Contreras, L. Navarro, G. Guardia, W. Guerra, D. Ruiz, y C. Gelsi.

2003. Análisis del material rescatado en Horco Molle. Libro de Resúmenes VI Jornadas de Comunicaciones: 12 . Tucumán. 
Cremonte, M. B.

1996. Investigaciones arqueológicas en la Quebrada de la Ciénaga (Dpto. de Tafí, Tucumán). Tesis Doctoral. Universidad Nacional de La Plata, La Plata.

Etcheverre, P.

1976. Normas de Reconocimiento de suelos. INTA. Castelar. Buenos Aires.

Heredia, O. R.

1975. Investigaciones Arqueológicas en el sector meridional de las Selvas Occidentales. Revista del Instituto de Antropología 5: 73-132.

Joyce, A. y S. Johannessen.

1996. Abandonment and the production of archaeological variability at domestic sites. Abandonment of settlements and regions. Ethnoarchaeological and archaeological approaches (editado por B. C. Cameron y S. Tomka), pp. 138-153 New Directions in Archeology. Cambridge University Press. Cambridge.

Maldonado, M. G., L. del Valle Neder, J. Roldán, y M. M. Sampietro Vattuone.

2009. Procesos de formación de sitios en el sector meridional de las Selvas Occidentales (sitio STucYeb 2 "Horco Molle", Yerba Buena, Tucumán, Argentina). Anales de Arqueología y Etnología. En prensa.

Martínez, J., N. Olisewski, E. Ribotta y C. Sotelos.

1994. Primeras Investigaciones Arqueológicas de la REHM. Resúmenes del II Congreso Nacional de Estudiantes de Arqueología. Santa Fé.

Míguez, G. E.

2005. Dinámica local y relaciones interétnicas: nuevas investigaciones y replanteo de la problemática arqueológica del piedemonte de la Sierra de San Javier. Dpto. de Yerba Buena, Pcia. de Tucumán. Trabajo Final de la Carrera de Arqueología. Universidad Nacional de Tucumán, Tucumán.

Míguez, G. E. y M. Gramajo Bühler.

2007. Tallando en las tierras bajas de Tucumán: análisis de artefactos líticos de Horco Molle, Provincia de Tucumán. XVI Congreso Nacional de Arqueología Argentina, III: 165-170. Jujuy.

Mon, R. y L. E. Suayter.

1972-1974. Geología de la Sierra de San Javier (Provincia de Tucumán, República Argentina). Acta Geológica Lilloana 12: 157-168.

Núñez Regueiro, V. A. y J. García Azcárate.

1996. Investigaciones arqueológicas en El Mollar, Departamento Tafí del Valle, Provincia de Tucumán. XI Congreso Nacional de Arqueología Argentina (13ª parte): 87-97. Mendoza.

Palamarczuk, V., R. Spano, F. Weber, D. Magnífico, S. López y M. Manasiewicz. 2006. Soria 2. Apuntes sobre un sitio formativo en el valle de Yocavil (Catamarca, Argentina). Intersecciones en Antropología $\mathrm{N}^{\mathrm{0}}$ 8: 121-135.

Primera Convención de Antropología, Primera Parte.

1966. I Convención Nacional de Antropología, Primera Parte. Instituto de Antropología, Publicaciones, Nueva Serie I (26). Córdoba. 
Ruiz, N. D.

2003. Análisis del ajuar de dos urnas funerarias. VI Jornadas de Comunicaciones: 27. Tucumán.

Ryden, $\mathrm{S}$.

1936. Archaeological research in the departament of Candelaria. Etnologiska Studier 3: 5-320.

Rye, O.S.

1981. Pottery technology, principles and reconstruction. Manuals of Archaeology 4, Taraxacum.

Sampietro Vattuone, M. M., J. m. Sayago, M. A. Caria, y M. M. Collantes.

2003. Cambios climáticos y dinámica poblacional en el noroeste argentino durante los períodos "Formativo" y "Desarrollos Regionales". Congreso Argentino de Cuaternario y Geomorfología, Acta II: 463-474.Tucumán.

Scattolin, M. C.

2007. Santa María antes del año mil. Fechas y materiales para una historia cultural. Sociedades Precolombinas Surandinas. Temporalidad, Interacción y Dinámica Cultural del NOA en el Ámbito de los Andes Centro-Sur (editado por V. I. Williams, B. N. Ventura, A. B. M. Callegari y H. D. Yacobaccio), pp. 203-220, Buenos Aires.

Scattolin M. C., L. P. Domingorena, L. I. Cortéz, M. F. Bugliani, C. M. Calo, A. D. Izeta y M. Lazzari.

2007 Cardonal: una aldea formativa entre los territorios de valles y puna. Cuadernos FHyCSUNJu, No 32: 211-225.

Schiffer, M.

1987. Formation processes of the archeological record. University of Utah Press. Salt Lake City.

Srur, F.

2001. La Cerámica de la Fase I de Tafí. En Actas del XIII Congreso Nacional de Arqueología Argentina (1999), Tomo 2: 180-192. Córdoba.

Tartusi M. R. A. y V. A. Núñez Regueiro.

2000. La presencia de Aguada en la Provincia de Tucumán. En "IV Mesa Redonda de la Cultura de La Aguada y su dispersión". S. P. de Atacama. www.geocities.com/ aguadamesaredonda/.

Van Zuidam R. y F. Van Zuidam,

1985. Terrain analysis and classification using aerial photograph. ITC, Textbook VII-6. Second Edition. The Netherlands.

Ventura, B. N.

2003. Apéndice. La Mitad Verde del Mundo Andino. Investigaciones arqueológicas en la vertiente oriental de los andes y las tierras bajas de Bolivia y Argentina, (editado por G. Ortíz y V. Ventura) pp. 319-323, Jujuy. 
